\title{
CHRONIC RHEUMATIC JOINT DISEASE IN GENERAL PRACTICE.*
}

\author{
By PHILIP ELLMAN, M.D., M.R.C.P.
}

(Consultant to the Rheumatic Unit at St. Stephen's Hospital (L.C.C.), Physician to the St. John Clinic and Institute of Physical Medicine (Order of St. John of Jerusalem), Etc.)

Chronic rheumatic joint disease is the greatest single cause of disability in temperate climates and is said to produce more pensionable invalidism than any other condition except cardiovascular disease in old age. It is therefore unfortunate that chronic arthritis, which is so formidable a scourge in this country, should, until recent years, have been relegated to the list of uninteresting and incurable diseases, and in consequence been treated almost with indifference by the profession as a whole. Because of the indifference shown by our profession to this group of diseases the growth of irregular practitioners has increased by leaps and bounds. Moreover, the indifference shown particularly to an important branch of therapeutics - "physical medicine" -in the treatment of this disease, has been largely responsible for the encouragement of such irregular practitioners, who have not failed to take real and unscrupulous advantage of our neglect.

\section{THE PROBLEM-A COMPARISON WITH TUBERCULOSIS.}

Like tuberculosis, rheumatic disease is a social and economic, as well as a medical problem, and the people so afflicted become a burden to their fellow citizens. From the clinical aspect, as will be shown when we come to discuss the infective or rheumatoid type of disease, chronic infective arthritis has, like tuberculosis, a general constitutional as well as a local disturbance. Both factors require adequate recognition in order to ensure successful treatment.

With a full knowledge of all that is involved in the adequate treatment of active pulmonary tuberculosis, which may be extended over months and even years, so in active arthritis the early recognition of an analogous time factor is essential if success is to be obtained. And further, in rheumatoid arthritis as in tuberculosis, quiescent disease may readily become reactivated unless adequate hygienic discipline is insisted upon. Also as in the case of tuberculosis, so in the chronic rheumatic diseases there is very real need for an active campaign in the form of state action against the existence of a curable disease which, if left untreated, results in permanent crippling and invalidism among every section of the community.

Sir George Newman, the late chief Medical Officer, has pointed out that "the chronic forms of rheumatism are very seriously crippling many hundreds and thousands of our people, who are not only thus subjected to suffering and disablement, but are losing heavily in time and money."

The need for state intervention as exists in tuberculosis will be appreciated if the investigations undertaken by the Ministry of Health in I924 and subsequently are examined. The report issued in that year was the first definite statement-in Great Britain or elsewhere-of the social burden of rheumatic 
disease in the light of insurance figures, and it stands as a landmark in the recognition of the serious social and economic aspects of these diseases. Sir Walter Kinnear's figures relating to the population under the National Health Insurance Scheme indicate that $£ \mathrm{I} 7,000,000$ represents payments under this scheme, and loss of wages due to rheumatism but exclusive of the cost of treatment among persons who, while suffering from rheumatism, are not totally disabled.

It has been calculated that rheumatic diseases account for one sixth of the industrial invalidity of Great Britain and as C. W. Buckley points out, the annual cost of $£ 20,000,000$ for their present treatment is probably too low an estimate. In spite of this vast expenditure on wholly preventable diseases, disablement still exists among the entire population, and there can surely be no doubt that the matter should be brought to the notice of local authorities and that state action-comprehending the establishment of clinics and sanatoria as in the case of tuberculosis-should be instituted.

In London, physical medicine, which is the most important therapeutic measure in the problem of rheumatism, is taken up seriously by the Order of St. John of Jerusalem, through the St. John Clinic and Institute of Physical Medicine in Pimlico, and by the British Red Cross Society at its clinics. The St. John Clinic, through the kind co-operation and interest of Sir Leonard Hill and Sir Frederick Menzies, has also the use of a valuable rheumatic unit at St. Stephen's Hospital (L.C.C.) where patients requiring institutional treatment can be accommodated.

It is obvious, however, that these few existing facilities touch only the fringe of the problem, but an investigation of the achievements so obtained indicates how valuable such institutions would prove if they were more generously provided throughout the country. The rheumatic problem should be attacked by the medical profession in the same way that tuberculosis has been attacked. Denmark and Sweden are among the countries which have faced it, but in England it has become a challenge to the whole profession. There must be a change of outlook which will recognize that a so-called chronic disease like rheumatism is, in fact, a very acute problem.

\section{CLASSIFICATION.}

Most unfortunately for the general practitioner, the clinical classification of chronic arthritis is confusing and chaotic. From an æetiological, pathological and clinical standpoint, as we shall see later, there are two distinct groups, which correspond to $(a)$ the so-called infective or rheumatoid group, and $(b)$ the osteo-arthritic group. Basing himself on the works of Nichols and Richardson, following pathological studies of chronic arthritis, of Herbert Assman, of Allison and Ghormley and of Timbrell Fisher, the writer has adopted a classification for clinical purposes which is comparatively simple and yet comprehensive. The two groups recognised are:-

(a) The atrophic or infective group of arthritis (corresponding to the socalled rheumatoid arthritis.)

(b) The hypertrophic or degenerative group (corresponding to the so-called osteo-arthritis.) 
Each group has, as a general rule, distinctive ætiology, pathology, symptoms and signs, although a mixed type of both atrophic and degenerative arthritis can and does occur.

\section{ETIOI.OGY.}

In the atrophic type, the joint changes, which are of an inflammatory nature, are essentially the result of some focal sepsis, which may be:-

(a) Oro-nasal, including teeth, tonsils, antrum or naso-pharynx.

(b) Alimentary, e.g., bowel, gall bladder or appendix.

(c) Urogenital, not excluding the significance of cervical infection in women and prostatic infection in men, as emphasized by Robinson.

In such cases there is a systemic infection analogous to that in tuberculosis, and the disease produces $(a)$ a general systemic disturbance, and $(b)$ local manifestations in affected joints.

In the hypertrophic or degenerative type, the principal rtiological factor is an endocrinal metabolic disturbance which occurs usually about the time of the climacteric.

Other factors, such as hereditary diathesis, constitution, body build, age, trauma, mental and physical strain associated with occupation, defective hygiene, climatic conditions and exposure to damp and cold, are, no doubt, common to both groups.

\section{PATHOLOGY.}

Each type of arthritis has its peculiar distinctive pathological charıges, and these are important from the point of view of diagnosis and treatment.

In the atrophic type, the lesion is essentially an inflammatory one, the synovial membrane being the first structure involved. The inflammatory process converts the synovial membrane into a highly vascular structure of granulation tissue. Subsequently a pannus of this tissue spreads over the joint cartilage and causes its erosion and destruction. A similar inflammatory change affects the endosteum of the epiphyseal bone marrow, invading the bone marrow, which is thus destroyed from above and below. Since the ends of the two articulating bones are thus destroyed and bridged over by granulation tissue, fibrous or even bony ankylosis may result, and this is generally associated with wasting of the neighbouring muscles. In advanced cases, the joint may be completely destroyed and the adjacent bones may become fused with a common bone marrow. The result is in many cases a secondary osteo-porosis which is well shown radiologically.

It should be mentioned that the synovial pannus may, and frequently does, extend into the periarticular tissues, producing the characteristic spindile-shaped deformity of the fingers. Synovial effusions are common. In view, therefore, of the possibility of ankylosis, regular movements of the joint must be encouraged through the whole duration of the disease.

In the degenerative or hypertrophic type, on the other hand, inflammation (apart from traumatic complications) is absent. The pathological changes are characterized by proliferation and degeneration of the hyaline cartilage of the articular surface, resulting finally in hypertrophy of the cartilage and bone of the 
joint. There is at first an irregular fibrillation of the cartilaginous matrix, and, as this becomes softened and eroded, it results in irregular exposure of the underlying bone which may itself eventually become eroded. On the opposing articular surfaces a compensatory overgrowth of cartilage and bone keeps the joint surfaces in contact with each other, although in an extremely irregular manner. This degenerative change may completely destroy the cartilage, bringing into contact the bones themselves, the articular surfaces of which may become very dense. The use and friction to which the bones become subjected gives them a high degree of polish (eburnation). This occurs only in degenerative arthritis, in which condition true ankylosis never occurs-hence movements of the joints in this type of disease do not arise-although locking and fixation of the joints from loose fringes of bone or cartilage may occur.

The distinctive ætiology and pathology described above justify the division of arthritis into two main groups, although as already mentioned, both types do occur at times in one and the same individual.

\section{SYMPTOMS AND SIGNS.}

In atrophic arthritis the onset may be either sudden or gradual and usually occurs in a young adult under forty. In addition to the general systemic disturbance, (malaise, lassitude, etc.), the disease may be ushered in by stiffness of one or more finger joints (usually the proximal interphalangeal) but any large joint or group of joints may be similarly involved, and the classical signs of inflammation may be present according to the acuteness of the inflammatory process. Pain and stiffness vary in duration and degree. Sometimes this may pass off in a day of two; at other times it may last for some time, and the patient, becoming acclimatised to the pain, unfortunately ignores it. Occasionally the process may be of a really acute nature, associated with general lymphatic glandular and sometimes even splenic enlargement. Muscle, nerve tissue and fibrous tissue may also be involved, and in these cases visceral disturbance is frequently simulated.

The patients usually look ill, wasted and toxic. They are of asthenic build. complain of being fatigued on the least effort, are irritable and nervous and show vaso-motor phenomena, e.g., poor circulation, cold extremities, bruising from 3 slight injury, and in some cases even Raynaud's disease. Hypochromic anæmia, psoriasis and other skin lesions are not infrequent accompaniments. The heart is rarely affected, unless there be rheumatic fever, although functional murmurs are common.

In women, there is frequently a history of illness dating from some pelvic mischief, or following a bad confinement or miscarriage. In addition to the above

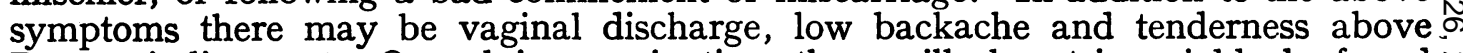
Poupart's ligament. On pelvic examination, there will almost invariably be found $\tilde{0}$ an endocervicitis. To use Robinson's description, there is, in fact, a characteristic $\underset{\omega}{\sim}$ "backache, cervicitis, arthritic syndrome." Constitutional symptoms in " atrophic arthritis" are thus seen to be well marked.

In striking contrast are the symptoms and signs of degenerative hypertrophic arthritis. The patient is usually robust, in the forty to sixty age period, and the mode of onset is insidious. The first joints to be involved are perhaps those of $\overrightarrow{\mathbb{D}}$ the knees, the patient complaining of stiffness after prolonged periods of either $\frac{\Omega}{\Phi}$ 
rest or movement. Later the joints become tender and there may be diffuse swelling. Unlike the toxic variety, effusion into the joint is quite uncommon and a characteristic grating crepitus may be both heard and felt, particularly in the knees. In the hands, the terminal phalangeal joints are usually involved, and sooner or later the characteristic swellings on one side of the joint-Heberden's nodes-are visible. These are in direct contrast to the fusiform swellings of the proximal phalangeal joints typical of atrophic arthritis. In many cases of degenerative arthritis the onset of symptoms is closely related to the climacteric (in men a " male climacteric" appears to occur some ten or fifteen years later, with symptoms of a less dramatic nature which are associated with prostatic deficiency). The patients frequently complain of headaches, flushings, sweats, giddiness, etc. The presence of an ovarian deficiency disturbs the joint nutrition, and the association of an ovarian deficiency with an existing hypothyroidism and chronic arthritis of the degenerative type is receiving ever more recognition. Such cases frequently present stigmata of hypothyroidism, such as dry skin, myxœdematous pads of fat above the clavicles, on the inner side of the knees, on the outer side of the hips, etc., together with obesity and myxœdematous facies.

In the majority of my cases there has also been evidence of a high blood pressure with varying degrees of left ventricular hypertrophy and myocardial disease, as shown on clinical, X-ray and electro-cardiographic examination. There is, therefore, an endocrinal metabolic disturbance, and accordingly a characteristic syndrome of " endocrinal arthritis-high blood pressure-and obesity" has come to be recognised.

\section{X-ray Appearances.}

In the early stages of atrophic arthritis there are often few if any abnormal radiographic joint changes in bone or cartilage. Where the peri-articular swelling is marked it is generally apparent and any thickening of the capsule or synovial membrane is often noticeable. Again, when bony atrophy begins, rarefaction is to be seen, while cartilage erosion shows itself by a narrowing of the joint space. As destruction of the cartilage continues, this space disappears completely and the articular bony surfaces come together.

Osteo-arthritis is characterised by degenerative changes in the cartilage and bone-hence the term "arthrosis" is sometimes applied. Radiograms of such cases reveal chondro-osseous ridges of the articular margins, deformations of articular bone ends by bony overgrowths, disappearance of articular cartilage, eburnation of articular surfaces of bone and the occurrence of loose bodies in the joint-" joint mice."

\section{Blood Changes.}

Blood investigations in the atrophic type of arthritis often show mild anæmia of the hypochromic variety, and the sedimentation rate of the red blood corpuscles is noticeably higher.

In hypertrophic arthritis the blood presents a normal picture and anæmia is rare. The blood sedimentation rate is, as a general rule, normal, but in many cases the blood uric acid is slightly raised. 
The main features of the two types of the disease are tabulated below:-

\begin{tabular}{|c|c|c|}
\hline & $\begin{array}{l}\text { Atrophic OR Proliferative } \\
\text { (inflammatory) }\end{array}$ & $\begin{array}{c}\text { DEGENERATIVE } \\
\text { (non-inflammatory) }\end{array}$ \\
\hline Principal ætiological factors & Focal sepsis & Endocrine disturbance \\
\hline Average age of onset & 20 to 40 & 40 to 60 \\
\hline Type of patient & Asthenic build & Robust build (obesity) \\
\hline Mode of onset & Acute or gradual & Gradual \\
\hline Infection & Focal sepsis usually present & May be absent \\
\hline Joints involved & $\begin{array}{l}\text { Any joint in the body; } \\
\text { spindle shaped swelling } \\
\text { of proximal interphalan- } \\
\text { geal joints of hands are } \\
\text { common }\end{array}$ & $\begin{array}{l}\text { Knees and fingers, He- } \\
\text { berden's nodes common }\end{array}$ \\
\hline Bones & Associated osteoporosis & $\begin{array}{l}\text { Condensation of articular } \\
\text { margins }\end{array}$ \\
\hline Muscular wasting & May be marked & Wasting from disuse \\
\hline Pain & Often marked & Not usually marked \\
\hline Ankylosis & Fibrous or bony may occur & Never occurs \\
\hline Constitutional symptoms & Often marked & Usually absent \\
\hline \multirow[t]{2}{*}{ Circulatory phenomena } & $\begin{array}{l}\text { Poor circulation of hands } \\
\text { and feet. Raynaud's } \\
\text { disease may occur }\end{array}$ & High blood pressure \\
\hline & $\begin{array}{l}\text { Hypochromic anæmia } \\
\text { common }\end{array}$ & $\begin{array}{l}\text { Hypochromic anæmia un- } \\
\text { common }\end{array}$ \\
\hline Skin diseases & $\begin{array}{l}\text { Psoriasis and other skin } \\
\text { conditions common }\end{array}$ & Very rare \\
\hline
\end{tabular}

TREATMENT.

The necessity for complete general medical examination before any treatment is begun cannot be over-emphasised. The physician must give to the general constitutional aspect of the case as much attention as to the local joint manifestations. No form of treatment can be applied indiscriminately to all cases, and many so-called "rheumatic" pains are secondary to diseases other than those of the articular system-more particularly the central nervous, cardiovascular and respiratory systems; e.g., cases of hysteria, tabes and disseminated sclerosis; aortic aneurysm with associated intercostal neuralgia; true pleuritic pain and pressure from new growths with secondary neuritis (carcinoma recti as the cause of sciatic pain may here be recalled).

\section{Psychological Considerations.}

As in all chronic diseases the psychological factor is not lacking in significance. I believe that the physician should acquaint the arthritic patient with the problems that face him, so that he may have a better understanding of the subject of the disease of which he is a victim. The physician, moreover, will, after making a careful enquiry into the history, learn something of the individual 
psychology of the patient, whilst simple methods of psycho-therapy, such as persuasion and suggestion, may change a hitherto pessimistic to a new optimistic outlook concerning treatment. So many patients are depressed by results of previous cases they have known-cases based on treatment by bad and obsolete methods-that they form the impression they are suffering from an incurable disease. Inadequate treatment does indeed often lead to contractures, ankylosis, muscular atrophy, circulatory disturbance and perhaps even confinement to a wheel-chair or bed; but, given early cases and accurate diagnosis, there are few diseases more amenable than chronic arthritis to adequate treatment.

\section{General Treatment.}

In all infective diseases everything must be done to bring the patient to the highest possible degree of physical fitness. The intelligent use of graduated rest and exercise in a suitable environment, together with the intelligent removal of focal sepsis, especially in the atrophic form, and the avoidance of unnecessary worry should help appreciably. The general treatment of the atrophic form is, in point of fact, analogous to that of pulmonary tuberculosis, and there is no doubt that many cases treated in institutions run on sanatorium lines derive immense benefit therefrom.

In all cases where constipation and bowel sepsis exist, intestinal antiseptics and change of the intestinal flora may be tried; while plombieres douches; correctly and carefully given-sometimes combined with colonic massage-have often produced very favourable results.

In women the examination of the cervix should be a routine procedure, since cervical sepsis is so often present and yet so rarely treated, although this neglect has frequently led to the wholesale extraction of teeth, tonsils, etc., without any effect whatsoever.

\section{Diet.}

There is no specific diet in arthritis, though belief in such a diet is common. The essential is that it should be well balanced with a limited amount of starches, sweets and meat. In atrophic arthritis a well balanced and nutritious diet with a high vitamin content comprising adequate quantities of protein and fats with carbohydrates answers the problem.

In degenerative arthritis where the patient is usually a robust individual the diet should be reduced both in quantity and quality. The physician should aim at a sub-caloric diet, low in carbohydrates, yet satisfying by reason of its bulk. Thus, as in diabetes, the meal should comprise a liberal supply of vegetables. For the average person of good nutrition, under conditions of complete or partial rest, 30 calories per kilo of body weight may be allowed, so that a diet varying from $I, 500$ to 2,000 calories a day is usually quite adequate.

\section{Drug Treatment.}

In the degenerative variety with metabolic disturbances, thyroid and ovarian extracts may be used with advantage. Iodine is also valuable in this group, particularly where co-existing cardiovascular changes are found. A mixture such as the following is often beneficial:-

Pot. Iod. grs. V.

Pot. Brom. grs. X.

Inf. Gent. Co. ad I oz. t.i.d.p.c. 
Tonic treatment with iron and arsenic, by mouth or intramuscularly, may sometimes be of advantage to the toxic group when it is associated, as it so frequently is, with a secondary anæmia. In certain cases, liver extract with iron has proved beneficial.

Sulphur. It has for some time been supposed that there is a disturbance of sulphur metabolism in chronic rheumatoid arthritis. Cawadias has been one of the foremost exponents of this view. There is little doubt that intramuscular injections of sulphur in selected cases has what would appear to be a specific effect. The use of sulphur by intramuscular injection certainly does have a beneficial effect in a number of cases in which it has been tried by us. Whether the effect be specific or non-specific it is valuable in that it produces fever therapy, especially when used in the form of the preparation Sulfosin (sulphurated oil). The method is safe and the dosage is not complicated. Its introduction intramuscularly produces a brief pyretic reaction. I use for preference a preparation sulfosin anæstheticum (I\% sulphur-a $2 \%$ sulphur solution can be used if stronger reactions be desired). Before use the preparation is heated and shaken, in order to dissolve the sulphur and the injection is made deep into the buttock. The temperature rises from $100^{\circ} \mathrm{F}$. to $103^{\circ} \mathrm{F}$. in about twelve hours and subsides in about twentyfour hours. Injections should be given twice weekly, starting with $\frac{1}{2}$ c.c. and working up to about 5 c.c., the number of injections varying from six to twelve in one course.

Insulin. Insulin, given in carefully graduated doses, can be used to great $\vec{\oplus}$ advantage where wasting and anorexia are features of the disease, as in certaing cases of hyperthyroidism and pulmonary tuberculosis. There is, to my know ledge, no better remedy for stimulating the appetite, when all the usual methods have failed, than a hypodermic injection of insulin. With improvement of appetite, the patient gains in weight and, still more important, his resistance is improved. The insulin in these cases appears to act as a metabolic fillip. Ordinarily, I start by giving the patient five units of insulin per day for one week, the injection being given 20 minutes before the principal meal and followed three hours later by glucose or a glass of milk, to avoid the risk of hypoglycæmia. The dose of insulin is increased each week by 5 units to a maximum of 30 units a day, given usually in two doses of $I_{5}$ units each. The patients are taught to give the injections themselves, just as diabetics do. In carefully selected cases insulin has proved to be a valuable systemic remedy. Cod liver oil and malt is also beneficial with the thin type of patient with vasomotor changes.

Gold. The first known instance of treatment with gold in modern times was in I9I2, when Bruck and Glück used it for cases of lupus erythematosus. Twelve years later Möllgard introduced Sanocrysin (gold sodium thiosulphate) for the treatment of pulmonary tuberculosis, and in I926 Feldt and others used it in carefully controlled experiments on dogs and mice infected with syphilis, spirochæta recurrens and streptococci, the effect of which showed it to have definite curative powers. In I929 Umber and Forrestier published the results of treatment of infective arthritis with gold compounds.

Up to the present we have treated some forty cases of infective arthritis, sixty per cent. having been treated with solganol B. (aurothioglucose), the remainder receiving injections of almond oil made up to simulate the gold preparation so that any psychic effect could be eliminated. All the patients had shown no definite improvement under other methods of treatment. 
In both groups the injections had a beneficial effect on the pain and stiffness, but the gold cases profited to a much greater degree than the controls treated with almond oil. Moreover, while three of the gold cases have been completely rid of pain and stiffness, not one of the controls has so far been cured.

The contrast in the effect on the sedimentation rate was striking. In the gold cases it sank after about two months to normal levels, where it remained, although there was sometimes a slight preliminary rise during the first month. In the controls, on the other hand, the sedimentation rate rose and fell, and on the rare occasions when it reached normal levels it remained there only temporarily.

Toxic effects were frequent in the cases treated with gold-erythema and morbilliform rashes, stomatitis, conjunctivitis and exfoliative dermatitis. One case developed agranulocytosis with purpura hæmorrhagica. Eosinophilia at the time of the toxic phenomena was a common occurrence.

The preparation used has been for the most part solganol B oleosum (solganol B suspended in oil) introduced intramuscularly into the buttock. The dosage is weekly injections of solganol B oleosum, commencing with $0.01 \mathrm{gm} ., 0.05 \mathrm{gm}$., O.I gm., the last dose being repeated until $2 \mathrm{gm}$. are given in all. Frequently a second course is given after an interval of six weeks.

\section{Vaccine Therapy and Non-Specific Protein.}

Whilst my own experience of vaccine therapy, even where autogenous vaccines have been carefully prepared by expert bacteriologists, has been unsatisfactory, I am aware that certain observers still have great faith in this form of treatment. In no case, however, should the use of vaccines serve as a substitute for the removal of toxic foci.

Sometimes non-specific protein in certain acute forms of arthritis is recommended, and T.A.B. vaccine, milk, peptone, etc., have all been used with varying degrees of success.

\section{Physical Methods.}

It is because of the supreme importance of physical methods in the treatment of all forms of chronic arthritis that I have left this form of therapy to the last. I should like to draw attention to the indifference such methods have received in the past in contrast to the great value that is attached to them to-day. Particularly noteworthy is the skin mechanism and its sluggish and inefficient action in chronic arthritis, while from the point of view of treatment the influence of heat when applied to the skin and vaso-dilator effect on its wide blood supply are very significant. The biochemical effect on the tissue cells is also very important. Finally, the knowledge that in chronic arthritis the normally somewhat limited blood supply suffers further limitation from vaso-constriction suggests that any means of increasing the blood supply would be of value.

Heat applied generally acts systemically in that it improves the patient's circulation and metabolism, while when applied locally to the affected joint it induces a vaso-dilatation. Furthermore, when it is followed by efficient massage, there ensues a dilatation of the lymphatic eliminative channels, which in turn aids the removal of inflammatory or metabolic products.

Heat may be applied in a variety of way, both locally and generally, e.g., by hydrotherapy, light or electrical therapy. 
Hydrotherapy applied by a variety of baths above body temperature, acts in similar ways, whether used locally or systemically. It produces a pyretic action and increases the adaptive powers of an inactive skin, promoting elimination by sweat secretion, hyperæmia and vaso-dilatation.

Light therapy. From the general systemic point of view the carbon arc, tungsten and mercury-vapour light-baths each have their special indications and serve a useful purpose in stimulating the defensive mechanism of the body and raising the bactericidal power of the blood (as shown by Leonard Hill and Eidinow).

The use of local infra-red radiations to the affected joints induces hyperæmia and produces dilation of the eliminative blood and lymphatic channels.

Intra Pelvic Diathermy. This form of treatment was first introduced and advocated by C. A. Robinson and merits wide recognition in the treatment of arthritis, alike in the atrophic group where the cervix in women and the prostate in men are frequently the seat of sepsis, and in the degenerative (endocrinal) group, which is so often associated with ovarian and thyroid deficieny.

Intra-pelvic diathermy applied in the earlier stage to either group may completely restore joint function. Robinson found that when applied to the cervix diathermy produced a temperature very near $I I 5^{\circ}$ degrees. It arrests pelvic inflammation and, in toxic arthritis, relieves the metastatic effects. In degenerative, menopausal arthritis, heating the ovaries counteracts existing endocrine deficiency, and by general vaso-dilatation induced by the heat it reduces the common complication of hypertension. In certain cases Robinson applies similarly ionization to the cervix.

\section{Massage and Manipulative Treatment.}

The use of massage with passive and active movements following heat has a definite place in the treatment of certain cases of chronic arthritis, and its results (where the masseur is fully cognizant of what is aimed at) are extremely valuable. It is essential, however, that he should co-operate closely with the physician. In cases unassociated with deformity, the physical methods already outlined pave the way for massage - which further assists elimination of diseased products-and if the joint is put through its full range of movement daily it will prevent stiffness and the formation of adhesions. In cases of atrophic arthritis this is particularly important, for here graduated movements of the involved joints must, so far as possible, be begun at the very start in order to avoid risk of fibrous or bony ankylosis.

In chronic cases of rheumatic joint deformities, the close co-operation of physician, physiotherapist and manipulative orthopædic surgeon is invaluable. The ordinary physical methods should first be given full trial and often they can remedy deformity and restore joint mobility without further aid. In any case they serve as a useful preliminary for more active measures.

A joint which shows signs of active mischief (e.g., heat, painful swelling or acute reaction after movements) should in no circumstances be manipulated, and should have have been free from such sign for several months before the adoption of this therapeutic measure. Accurate diagnosis to dismiss tuberculous disease is essential. 
Where there are adhesions, or where there is stiffness of moderate or even advanced degree, an expert may give progressive manipulation, with intervening stages when the appropriate physiotherapeutic methods are used. Sometimes the manipulation may have to be done under general anæsthesia and this should not be discouraged. On the contrary, it is fortunate that the value of such treatment, where ordinary physiotherapeutic methods have been unavailing, should be receiving ever increasing recognition. For a detailed account of manipulative methods in chronic arthritis reference should be made to the works of A. G. Timbrell Fisher.

\section{X-ray Therapy.}

The recent application of this form of treatment in chronic arthritis is worthy of note, and in certain cases of degenerative arthritis, localized more particularly to hips and spine, it is especially valuable. It is said that the X-rays are analgesic, reduce congestion and cause absorption of pathological fibrous tissue. A number of striking results have been reported yet it is questionable what percentage would not have responded to the usual physiotherapy methods.

In conclusion I should like, however, to emphasize that, whilst certain general principles must necessarily be adopted in the treatment of arthritis, no single form of treatment can be applied to all cases and sometimes an intelligent combination of all forms, including possibly manipulation, may be required. In addition, any treatment which disregards the systemic nature of the disease will, at least in atrophic arthritis, inevitably meet with failure.

\section{CONCLUSIONS.}

I. Chronic rheumatic joint disease from an ætiological, pathological, clinical and radiological standpoint can be classified into two group:(a) atrophic (corresponding to the so-called rheumatoid or infective arthritis) and (b) degenerative or hypertrophic (corresponding to the so-called osteo-arthritis).

2. The joint changes are essentially inflammatory in the one group and noninflammatory in the other group.

3. A mixed type of arthritis, i.e., the co-existence of atrophic (proliferative) and degenerative arthritis, may occur in the same patient.

4. The local and systemic nature of the disease must be recognized.

5. The disease is preventable and its cure depends upon early diagnosis and efficient treatment.

6. Physical medicine plays an all-important part in the treatment of chronic arthritis.

7. Successful treatment is dependent upon a recognition of the ætological factor. 